\title{
Structural Cues Involved in Endoplasmic Reticulum Degradation of G85E and G91R Mutant Cystic Fibrosis Transmembrane Conductance Regulator
}

\author{
Ximing Xiong, ${ }^{*}$ Alvina Bragin, ${ }^{*}$ Jonathan H. Widdicombe, ${ }^{\ddagger}$ Jonathan Cohn, ${ }^{\S}$ and William R. Skach* \\ *Department of Molecular and Cellular Engineering and Department of Medicine, University of Pennsylvania, Philadelphia, \\ Pennsylvania 19104; ${ }^{\ddagger}$ Cardiovascular Research Institute, University of California, San Francisco, California 94143; and ${ }^{\S}$ Department of \\ Medicine, Duke University, Durham, North Carolina 27514
}

\begin{abstract}
Abnormal folding of mutant cystic fibrosis transmembrane conductance regulator (CFTR) and subsequent degradation in the endoplasmic reticulum is the basis for most cases of cystic fibrosis. Structural differences between wild-type (WT) and mutant proteins, however, remain unknown. Here we examine the intracellular trafficking, degradation, and transmembrane topology of two mutant CFTR proteins, G85E and G91R, each of which contains an additional charged residue within the first putative transmembrane helix (TM1). In microinjected Xenopus laevis oocytes, these mutations markedly disrupted CFTR plasma membrane chloride channel activity. G85E and G91R mutants (but not a conservative mutant, G91A) failed to acquire complex $N$-linked carbohydrates, and were rapidly degraded before reaching the Golgi complex thus exhibiting a trafficking phenotype similar to $\Delta$ F508 CFTR. Topologic analysis revealed that neither G85E nor G91R mutations disrupted CFTR $\mathrm{NH}_{2}$ terminus transmembrane topology. Instead, WT as well as mutant TM1 spanned the membrane in the predicted C-trans (type II) orientation, and residues $85 \mathrm{E}$ and $91 \mathrm{R}$ were localized within or adjacent to the plane of the lipid bilayer. To understand how these charged residues might provide structural cues for ER degradation, we examined the stability of WT, G85E, and G91R CFTR proteins truncated at codons 188, 393, 589, or 836 (after TM2, TM6, the first nucleotide binding domain, or the $\mathrm{R}$ domain, respectively). These results indicated that G85E and G91R mutations affected CFTR folding, not by gross disruption of transmembrane assembly, but rather through insertion of a charged residue within the plane of the bilayer, which in turn influenced higher order tertiary structure. (J. Clin. Invest. 1997. 100:1079-1088.) Key words: CFTR • cystic fibrosis • protein folding $\bullet$ protein biogenesis $\bullet$ ER degradation
\end{abstract}

\section{Introduction}

Cystic fibrosis $(\mathrm{CF})^{1}$ is caused by diverse mutations in the cystic fibrosis transmembrane conductance regulator (CFTR), a polytopic integral membrane protein expressed at the apical

Address correspondence to Dr. William R. Skach, Department of Molecular and Cellular Engineering, University of Pennsylvania, Philadelphia, PA 19104. Phone: 215-898-0953; FAX: 215-573-8590.

Received for publication 31 March 1997 and accepted in revised form 24 June 1997.

J. Clin. Invest.

(c) The American Society for Clinical Investigation, Inc. 0021-9738/97/09/1079/10 \$2.00

Volume 100, Number 5, September 1997, 1079-1088

http://www.jci.org membrane of selected epithelial tissues (1-3). CFTR is a member of the ATP Binding Cassette transporter superfamily (4, 5 ), and is proposed to function as a cAMP-regulated transmembrane channel capable of conducting small anions, water and ATP (6-9). More than 700 different disease-related mutations that affect different aspects of CFTR function have been reported throughout the CFTR gene $(10,11)$. In greater than $80 \%$ of CF patients, however, disease results from a temperature-sensitive folding defect (12) in which newly synthesized CFTR protein fails to process normally, and undergoes rapid degradation before reaching the plasma membrane (13).

Like most polytopic proteins, CFTR synthesis occurs in the rough endoplasmic reticulum (ER) where early events of folding and assembly take place. Both wild-type (WT) and certain mutant CFTR proteins may mature sufficiently during this process to acquire normal gating and ion channel properties while still in the ER membrane (14). CFTR exit from the ER, however, is quite inefficient; $\sim 80 \%$ of WT and up to $100 \%$ of mutant (e.g., $\Delta$ F508) CFTR may be degraded before reaching the Golgi complex $(12,13,15,16)$. Retention of CFTR in the ER induced by Brefeldin A or block of CFTR degradation with proteasome inhibitors has indicated that degradation of CFTR per se is not the primary defect (15-17). Rather, degradation appears to result from the failure of newly synthesized chains to acquire the proper structure necessary for exiting the ER. Recent studies have therefore suggested that a critical maturation step (or alternatively a misfolding step) occurs during or very early after CFTR synthesis in the ER, and determines whether newly synthesized chains $(a)$ mature into a normally folded, transport-competent structure, or $(b)$ misfold into a transport-incompetent structure that is retained and degraded $(15,18)$. Consistent with this hypothesis, $\triangle$ F508 CFTR exhibits prolonged association with cytosolic and ER chaperones, hsp70 (19) and calnexin (20), respectively, that recognize and bind misfolded or partially folded nascent proteins. In addition, chemical chaperones such as glycerol and trimethyl amineoxide, which may facilitate protein folding, have been shown to promote $\Delta$ F508 CFTR trafficking to the plasma membrane $(21,22)$. Despite these and additional studies $(23$, 24), structural differences between WT and mutant CFTR, and hence the mechanisms by which these proteins are distinguished by ER quality control machinery, remain unknown.

Significant evidence indicates that transmembrane topology of polytopic proteins is directed through the action of discrete sequence determinants (i.e., topogenic sequences [25]) encoded within the nascent chain (26-30). These determinants

1. Abbreviations used in this paper: $\mathrm{CF}$, cystic fibrosis; $\mathrm{CFTR}$, cystic fibrosis transmembrane conductance regulator; ER, endoplasmic reticulum; MBS, modified Barth's solution; MDRI, human P-glycoprotein; NBD, nucleotide binding domain; PK, proteinase K; RRL, rabbit reticulocyte lysate; TM, transmembrane; WT, wild-type. 
are comprised of hydrophobic transmembrane segments along with their flanking sequences (31), and function to direct translocation of specific peptide regions across the ER membrane. Recently, topogenic sequences encoded within the first two transmembrane segments (TM1 and TM2) of human P-glycoprotein (MDR1) were shown to direct transmembrane topology and membrane integration in a cooperative manner (32). These results defined MDR1 $\mathrm{NH}_{2}$ terminus topology, and confirmed that TM1 and TM2 spanned the ER membrane in their predicted orientation. Using similar techniques, we have begun to investigate the transmembrane topology of CFTR to better understand the relationships among CFTR folding, transmembrane assembly, and intracellular trafficking.

In the current study, we have examined the effects of two CF-related mutations, G85E and G91R, each of which introduces a charged residue into the first putative transmembrane helix (TM1) of CFTR $(33,34)$. These mutations markedly impaired CFTR-mediated chloride channel activity in the plasma membrane of microinjected Xenopus oocytes, and like $\Delta \mathrm{F} 508$, resulted in rapid degradation of newly synthesized protein in a preGolgi compartment. Remarkably, neither mutation altered the transmembrane topology of CFTR in the ER membrane, indicating that mutant residues were localized within or adjacent to the plane of the lipid bilayer. Furthermore, oocyte degradation machinery was unable to distinguish WT from G85E or G91R mutant proteins until synthesis of the first half of CFTR (through the first nucleotide binding domain [NBD1]) was completed. These findings suggest that insertion of a charged residue within (or adjacent to) the plane of the lipid bilayer at position $85 \mathrm{E}$ or $91 \mathrm{R}$ disrupts CFTR intracellular trafficking, not by gross disruption of transmembrane topology, but rather through subtle perturbations in tertiary structure.

\section{Methods}

cDNA construction. G85E and G91R mutants were synthesized from plasmid pSPCFTR (7) by site-directed mutagenesis using a singlestranded (M-13) template, oligonucleotides GTTCTATGAGATCTTTT or TATATTTAAGGGAAGTC, and T4 polymerase by the Muta-gene (Bio-Rad Laboratories, Hercules, CA) method. Ava I-Xba I fragments containing the engineered mutations were then ligated back into pSPCFTR (from which a $3^{\prime}$ untranslated Sac I fragment was deleted for convenience) and sequenced from the initial ATG through the Xba I fusion site. G91A and G91E mutants were synthesized by ligating an Ava I-Xba I fragment from plasmids pBQ4.7 G91A and pBQ4.7 G91E (kindly provided by D. Dawson, University of Michigan), respectively, into Ava I-Xba I-digested pSPCFTR; mutations were verified by DNA sequencing. To generate plasmids TM1-2.P, WT, G85E, G91R, G91A, or G91E, CFTR cDNA was amplified using 12 cycles of PCR (sense oligonucleotide ATTTAGGTGACACTATAG [SP6 promoter], antisense oligo AAATTTGGTCACCTTGTTGGAAAGGAGACT). Fragments were digested with Hind III/BstE II (site encoded in antisense oligo), and ligated into a Hind III/BstE II digested vector onto the $5^{\prime}$ end of a previously described prolactin-derived reporter sequence $(\mathrm{P})$ in plasmid BPI (32). Resultant plasmids contain CFTR codons M1-N148 followed by the $\mathrm{COOH}$ terminal 142 codons of prolactin (30). Plasmid TM1-6.P, encoding CFTR residues 1-392, was similarly constructed from WT, G85E, and G91R CFTR templates using the sense and antisense oligos ATTTAGGTGACACTATAG and ATTCTCGGTCACCACTTCTGTAGTCGTTAA, respectively. A termination codon was engineered following TM2 and TM6 by digesting WT, G85E and G91R, TM1-2.P, or TM1-6.P with BstE II, blunting ends with Klenow, and religating. This introduces an in-frame TGA codon immediately behind the BstE II restriction site. A TGA condon was engineered at codon 589 of WT, G85E, and G91R CFTR by ligating an Sph I/Pst I-digested PCR fragment (sense oligo ATCAGACCATGGGGAAGATCAGTGAAAG, antisense oligo AGTTTCTGCAGCTAGCTTTCAAATATTTCTT, template pSPCFTR) into a pSPCFTR plasmid digested with Sph I/Pst I. A similar strategy was used to engineer a TAG stop codon at residue D836. A CFTR PCR fragment was amplified using sense (ATCAGACCATGGGGAAGATACAGTGAAAG) and antisense (CTCGAATTCTAATCAAAAAGGCACTCCT) oligonucleotides, digested with EcoR I, and ligated into plasmid pSPCFTR digested with EcoR I.

Xenopus oocyte Cl transport. CFTR-mediated ${ }^{36} \mathrm{Cl}$ efflux was measured as described previously (9). In brief, oocytes were injected with in vitro-transcribed RNA and incubated at $18^{\circ} \mathrm{C}$ for $36 \mathrm{~h}$ in MBS (modified Barth's solution [35], $88 \mathrm{mM} \mathrm{NaCl}, 1 \mathrm{mM} \mathrm{KCl}, 2.4 \mathrm{mM}$ $\mathrm{NaHCO}_{3}, 0.82 \mathrm{mM} \mathrm{Mg} \mathrm{SO}, 0.33 \mathrm{mM} \mathrm{Ca}\left(\mathrm{NO}_{3}\right)_{2}, 0.41 \mathrm{mM} \mathrm{CaCl}_{2}, 10$ $\mathrm{mM}$ sodium $N$-2-hydroxyethylpiperazine- $N^{\prime}$-2-ethanesulfonic acid [Hepes]) followed by incubation overnight in MBS containing 20-40 $\mu \mathrm{Ci} / \mathrm{ml}{ }^{36} \mathrm{Cl}$ (total $\mathrm{Cl}$ concentration adjusted to $90 \mathrm{mM}$ ). Oocytes were then rinsed, and groups of five oocytes were perfused $(1 \mathrm{ml} / \mathrm{min})$ with MBS. Perfusate was collected at 1-min intervals, and radioactivity was determined by scintillation counting. After $8 \mathrm{~min}$, the perfusate was changed to MBS containing $1 \mathrm{mM}$ 3-isobutyl-1-methylxanthine and $10 \mu \mathrm{M}$ forskolin. At the end of the experiment, oocytes were dissolved in $1 \mathrm{~N} \mathrm{NaOH}$, and the total counts remaining were determined. For each flux interval, the radioactivity in oocytes at the start of the interval was calculated, and the loss of ${ }^{36} \mathrm{Cl}$ was expressed as the fraction of total counts. To determine the maximal cAMP-induced increase in the rate of loss of ${ }^{36} \mathrm{Cl}$, the period of greatest fractional rate of loss (after stimulation) was averaged with the rates of loss for the single intervals before and after (if the rate of loss was greatest in the final flux period, the average of the penultimate and final flux intervals was used). The baseline rate of loss was taken as the average of the three intervals immediately before stimulation.

Antibodies. Antisera $\mathrm{A}_{2}$ and 5242 were raised in New Zealand White rabbits using synthetic peptides corresponding to CFTR residues no. 45-65 (Berkeley Antibody Company, Berkeley, CA) or residues no. 2-16 (Research Genetics, Huntsville, AL), respectively. $\alpha-102$ antisera was raised against a CFTR synthetic peptide (residues no. 102-116) as reported previously (36). Antiprolactin antiserum was purchased from ICN Biomedicals, Inc. (Costa Mesa, CA).

Transcription and Xenopus laevis oocyte expression. mRNA was transcribed in vitro with SP6 RNA polymerase (New England Biolabs Inc., Beverly, MA) using 2-4 $\mu$ g of plasmid DNA in a $10-\mu \mathrm{l} \mathrm{vol}$ at $40^{\circ} \mathrm{C}$ for $1 \mathrm{~h}$ as previously described (30). $50 \mu \mathrm{Ci}$ of ${ }^{35} \mathrm{~S}$ methionine $\left(0.5 \mu \mathrm{l}\right.$ of a $10 \times$ concentrated $\operatorname{Tran}^{35} \mathrm{~S}$-label; New England Nuclear, Boston, MA) was added to $2 \mu \mathrm{l}$ of transcription mixture and injected into mature Xenopus oocytes (50 nl/oocyte) on an ice-cold stage. For pulse-chase experiments, injected oocytes were first incubated at $18^{\circ} \mathrm{C}$ in MBS and then transferred into MBS containing 1-2 mM unlabeled methionine. At specified times, oocytes (five per lane) were removed and frozen for later homogenization in $10 \mathrm{vol}$ of homogenization buffer ( $0.25 \mathrm{M}$ sucrose, $50 \mathrm{mM}$ Tris, $\mathrm{pH} 7.5,50 \mathrm{mM} \mathrm{KAc,} 5 \mathrm{mM}$ $\mathrm{MgCl}_{2}, 1 \mathrm{mM}$ DTT). Homogenates were mixed with $100 \mu \mathrm{l}$ of $1 \%$ SDS, $0.1 \mathrm{M}$ Tris, $\mathrm{pH} 8.0$, incubated at $37^{\circ} \mathrm{C}$ for $30 \mathrm{~min}$, diluted in $1 \mathrm{ml}$ of Buffer A (0.1 M NaCl, 1\% Triton X-100, 2 mM EDTA, $0.1 \mathrm{mM}$ PMSF, and $0.1 \mathrm{M}$ Tris, $\mathrm{pH} 8.0$ ), incubated at $4^{\circ} \mathrm{C}$ for $2 \mathrm{~h}$, and centrifuged at $14,000 \mathrm{~g}$ for $15 \mathrm{~min}$. The supernatants were then used for immunoprecipitation.

Cell-free translation/protease digestion. Transcription reaction mixture was added to rabbit reticulocyte lysate (RRL) containing ${ }^{35} \mathrm{~S}$ methionine as described previously (32) and incubated for $1 \mathrm{~h}$ at $24^{\circ} \mathrm{C}$. Where indicated, canine pancreas microsomal membranes (final concentration of $8.0 \mathrm{OD}_{280}$ ) were added at the start of translation. $\mathrm{CaCl}_{2}$ $(10 \mathrm{mM})$ was added before proteolysis. Proteinase K (PK) $(0.2 \mathrm{mg} / \mathrm{ml}$ final concentration) was added to RRL in the presence or absence of $1 \%$ Triton X-100, and samples were incubated on ice for $1 \mathrm{~h} \mathrm{(32).} \mathrm{Re-}$ sidual protease was inactivated by rapid addition of PMSF $(10 \mathrm{mM})$ 
followed by mixing in $10 \mathrm{vol}$ of $1 \%$ SDS, $0.1 \mathrm{M}$ Tris, $\mathrm{pH} 8.0$ (preheated to $100^{\circ} \mathrm{C}$ ).

Immunoprecipitation and autoradiography. Antisera was added to translation mixtures diluted in $0.5 \mathrm{ml}$ of Buffer A or oocyte homogenate to a working dilution of 1:1000. After 10-30 min preincubation, $5 \mu \mathrm{l}$ of protein A Affigel (Bio-Rad) was added, and the sample was mixed at $4^{\circ} \mathrm{C}$ for $2-10 \mathrm{~h}$ before washing three times with Buffer $\mathrm{A}$ and twice with $0.1 \mathrm{M} \mathrm{NaCl}, 0.1 \mathrm{M}$ Tris, $\mathrm{pH}$ 8.0. For endoglycosidase digestion, washed protein A Affigel beads were incubated for $3 \mathrm{~h}$ at $37^{\circ} \mathrm{C}$ with $2 \mu \mathrm{l}$ of $0.1 \mathrm{M} \mathrm{Na}$ citrate, $\mathrm{pH} 5,0.1 \%$ SDS, and $500 \mathrm{U}$ endoglycosidase $\mathrm{H}$, or $1 \mu \mathrm{l} 0.5 \%$ SDS, $1 \% \beta$-mercaptoethanol, $0.5 \mathrm{M} \mathrm{Na}_{3} \mathrm{PO}_{4}, \mathrm{pH}$ $7.5,10 \% \mathrm{NP}-40$, and $500 \mathrm{U}$ PNGase F. Samples were analyzed by SDS-PAGE, EN ${ }^{3} \mathrm{HANCE}$ (New England Nuclear) fluorography, and autoradiography. Autoradiograms were digitized with an AGFA Studio Scan II and, where indicated, band intensities were quantified on unmodified images using Adobe Photoshop.

\section{Results}

Chloride channel activity of G85E and G91R CFTR. To determine whether G85E and G91R mutations disrupted CFTR chloride channel function, we expressed full-length wild-type and mutant proteins in mature Xenopus oocytes by RNA microinjection and measured channel activity using a radionuclide efflux assay. CFTR- or mock-injected oocytes were loaded with ${ }^{36} \mathrm{Cl}$ for $12-16 \mathrm{~h}$, and CFTR channel activity was determined by measuring the rate of ${ }^{36} \mathrm{Cl}$ efflux before and after elevation of intracellular cAMP. As shown in Fig. 1, oocytes expressing WT CFTR exhibited a rapid rise in chloride permeability after elevation of intracellular cAMP, whereas chloride permeability was not significantly altered in mockinjected oocytes. In contrast to WT CFTR, cAMP-stimulated chloride efflux rates measured in oocytes expressing G85E, G91R, or $\triangle$ F508 CFTR were all delayed and markedly attenuated. The maximal increase in ${ }^{36} \mathrm{Cl}$ efflux observed in oocytes expressing WT CFTR was $14.2 \pm 1.8 \% / \mathrm{min}(n=8)$. For G91R and G85E CFTR, the maximal increases were $1.1 \pm 0.4 \% / \mathrm{min}$ ( $n=5$, with one batch of oocytes not responding) and 1.8 \pm $0.4 \% / \mathrm{min}(n=7)$, respectively. Oocytes expressing $\Delta \mathrm{F} 508$ CFTR showed a maximal increase in ${ }^{36} \mathrm{Cl}$ efflux of $3.6 \pm 1.1 \%$ / $\min (n=5)$, or $\sim 15 \%$ of WT, in good agreement with previous studies (37). Whereas the maximal chloride efflux for WT CFTR was observed $8.5 \pm 1.0 \% / \mathrm{min}$ after cAMP stimulation, chloride efflux from oocytes expressing mutant proteins rose much more slowly and continued until the experiment was terminated 14 min after cAMP stimulation.

CFTR synthesis in Xenopus oocytes. Despite the widespread use of Xenopus oocytes in studying CFTR ion channel function $(37,38)$, little is known regarding CFTR synthesis, processing, and trafficking in these cells. To address this issue, we employed pulse-chase labeling techniques by coinjecting CFTR cRNA and ${ }^{35} \mathrm{~S}$ methionine into mature Xenopus oocytes followed by incubation in media containing excess unlabeled methionine. Using $\mathrm{NH}_{2}$ terminus peptide-specific CFTR antisera, we were first able to detect CFTR fragments 30-45 min after microinjection of cRNA. Synthesis of full-length CFTR protein, however, required 60-90 min (Fig. 2 A, arrow). Full-length CFTR chains initially migrated on SDS-PAGE as a relatively sharp band with an estimated molecular weight of $150 \mathrm{kD}$ (Fig. 2, $A$ and $B$, upward arrow). 12-48 h after injection, larger forms of CFTR were observed that migrated together as a broad band of $\sim 165 \mathrm{kD}$ (Fig. 2, $B$ and $C$, downward arrows). Neither of these bands was observed in mock- injected oocytes, nor in CFTR-injected oocytes immunoprecipitated with nonimmune or irrelevant antisera (data not shown). Incubating oocytes in media containing $1 \mathrm{mM}$ methionine immediately after injection effectively blocked incorporation of ${ }^{35} \mathrm{~S}$ methionine into CFTR (Fig. $2 \mathrm{~B}$ ). Digestion of immunoprecipitated products with endoglycosidase $\mathrm{H}$ (Endo $\mathrm{H})$ resulted in a $4-6 \mathrm{kD}$ shift in migration of the $150-\mathrm{kD}$ band, consistent with cleavage of 2 high mannose $N$-linked carbohydrates (Fig. 2 C, upward arrows). In contrast, the $165-\mathrm{kD}$ form of CFTR was resistant to Endo $\mathrm{H}$ digestion but was sensitive to digestion with $\mathrm{N}$-glycosidase $\mathrm{F}$, demonstrating that in these chains, core $N$-linked carbohydrates had undergone processing by glycosyltransferases in the Golgi complex. Thus, the 150-kD and 165-kD forms of CFTR generated in Xenopus oocytes appear to be analogous to the ER-glycosylated (band B) and Golgi-processed (band C) forms, respectively, which have previously been described in mammalian cells $(12,13)$.

It should be noted that the rates of synthesis and processing in oocytes were markedly slower than in mammalian cells $\left(18^{\circ} \mathrm{C}\right.$ versus $\left.37^{\circ} \mathrm{C}\right)$, presumably reflecting differences in metabolism and lower incubation temperatures. In addition, minor variations in the extent and efficiency of carbohydrate processing were also observed in oocytes from different frogs (data not shown).

Intracellular trafficking of G85E and G91R CFTR. We next tested whether G85E and G91R mutations affected protein expression, stability, and/or intracellular trafficking using pulse-

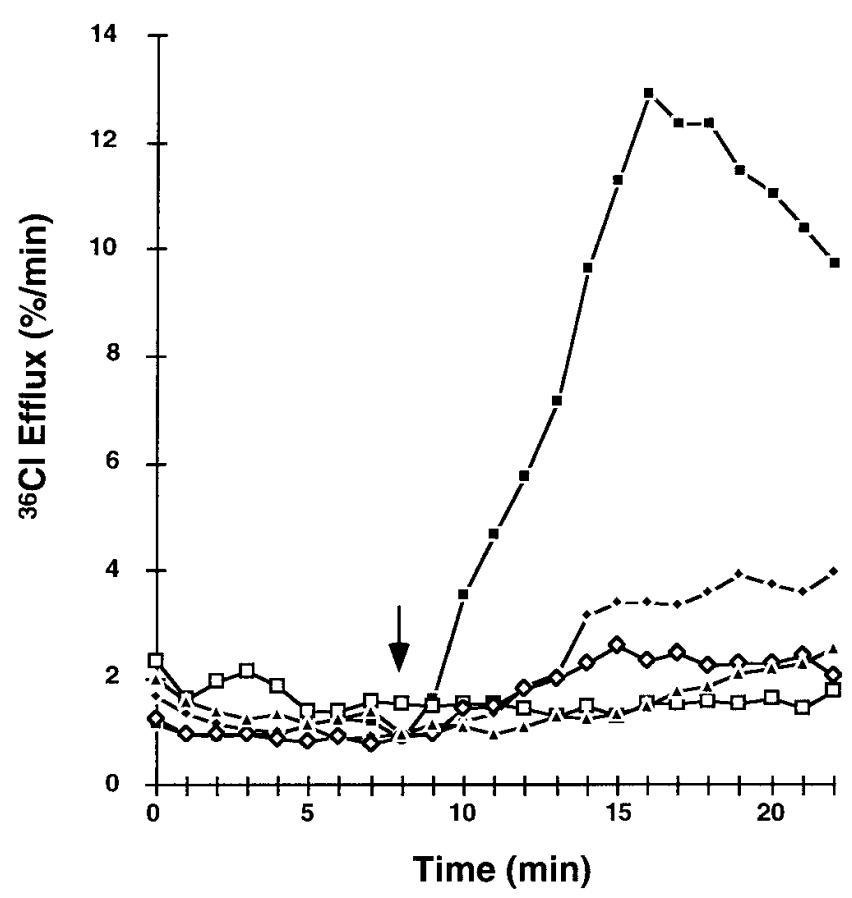

Figure 1. Function of WT and mutant CFTR. Effects of elevating cAMP on efflux of ${ }^{36} \mathrm{Cl}$ from mock, WT, $\Delta \mathrm{F} 508$, G85E, or G91R CFTR-injected oocytes. IBMX and Forskolin were added at $\mathrm{t}=8$ min (arrow) and were present continuously for the remainder of the experiment. Plotted are mean values: $N=8$, wild-type; $N=5$, G91R; $N=7$, G85E; and $N=5, \Delta$ F508. Standard errors averaged $\sim 15 \%$ of mean values, and have been omitted for clarity. Filled squares, wildtype; open squares, mock; closed diamonds, $\Delta \mathrm{F} 508$; open diamonds, G91R; and closed triangles, G85E. 


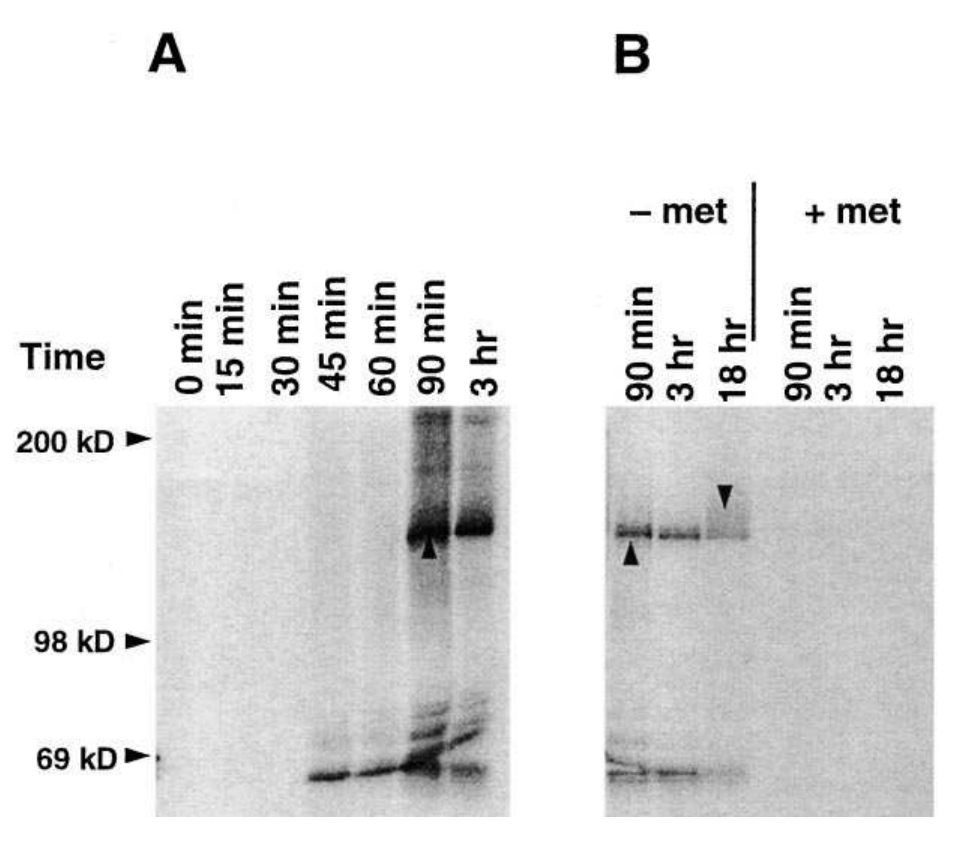

\section{C}

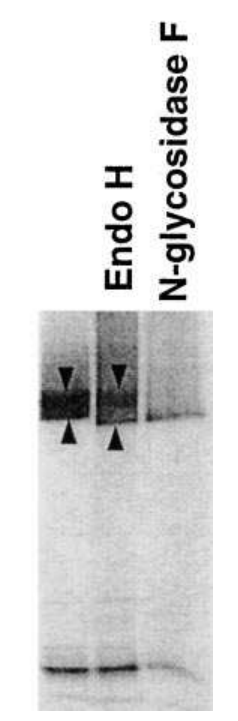

Figure 2. CFTR expression in Xenopus oocytes. $(A)$ Full-length WT CFTR cDNA was microinjected into Xenopus oocytes together with ${ }^{35} \mathrm{~S}$ methionine. At times indicated, oocytes were collected, homogenized, and immunoprecipitated with $\mathrm{A}_{2}$ antisera, and were analyzed by SDS-PAGE. (B) After injection, oocytes were immediately incubated in MBSH (-met) or in MBS containing $1 \mathrm{mM}$ unlabeled methionine $(+$ met $)$. At indicated times, oocytes were homogenized, immunoprecipitated with $\mathrm{A}_{2}$ antisera, and analyzed by SDS-PAGE. (C) Oocytes microinjected as in $A$ were incubated for $48 \mathrm{~h}$ in the absence of unlabeled methionine before homogenization and immunoprecipitation. Immunoprecipitates were digested with endoglycosidase $\mathrm{H}$ or $\mathrm{N}$-glycosidase $\mathrm{F}$ as indicated. All results shown are for five oocytes per lane. chase conditions described in Fig. 2. Oocytes were coinjected with cRNA and ${ }^{35} \mathrm{~S}$ methionine, and after $3.5 \mathrm{~h}$ were transferred to media containing $1 \mathrm{mM}$ unlabeled methionine before collection for immunoprecipitation. Similar amounts of WT and mutant CFTR were synthesized during the labeling period (Fig. 3). 24-30 h after injection, essentially $100 \%$ of WT CFTR was processed to the $165-\mathrm{kD}$ band $\mathrm{C}$ form (Fig. 3 A, downward arrow), indicating efficient protein trafficking through the Golgi complex. In contrast, G85E and G91R mutant proteins were rapidly degraded within $24 \mathrm{~h}$. Less than $10 \%$ of newly synthesized mutant protein was recovered $32 \mathrm{~h}$ after RNA injection (Fig. 3, $C$ and $D$ ), and less than $5 \%$ of mutant protein was processed to the $165-\mathrm{kD}$ band $\mathrm{C}$ form. Both the rapid disappearance and lack of Golgi processing strongly suggested that mutant proteins failed to exit the ER. Consistent with this interpretation, we observed that the known trafficking mutant $\Delta$ F508 CFTR also underwent rapid degradation (80-85\% of protein was degraded within $20 \mathrm{~h}$ ), similar to G85E and G91R chains (Fig. 3 B). A small but significant fraction of $\Delta$ F508 chains $(10 \%)$, however, was processed to a stable band $\mathrm{C}$ form (Fig. 3 B, downward arrow). While the absolute trafficking efficiency of WT and mutant CFTR varied somewhat between different batches of oocytes, selective degradation of mutant chains was consistent and reproducible. These experiments demonstrate that Xenopus oocytes, like mammalian cells, exhibit a quality control system that is capable of discriminating WT from mutant CFTR, and targeting mutant proteins for rapid degradation in the ER.

Effects of G85E and G91R mutations on CFTR topology. To determine whether G85E and G91R mutations altered the orientation of CFTR in the ER membrane, we compared the $\mathrm{NH}_{2}$ terminus topology of WT and mutant chains. A RRL translation system supplemented with ER-derived canine pancreas microsomal membranes was used for these experiments because this system has been shown to reconstitute faithfully ER translocation and early processing events (39), and it is

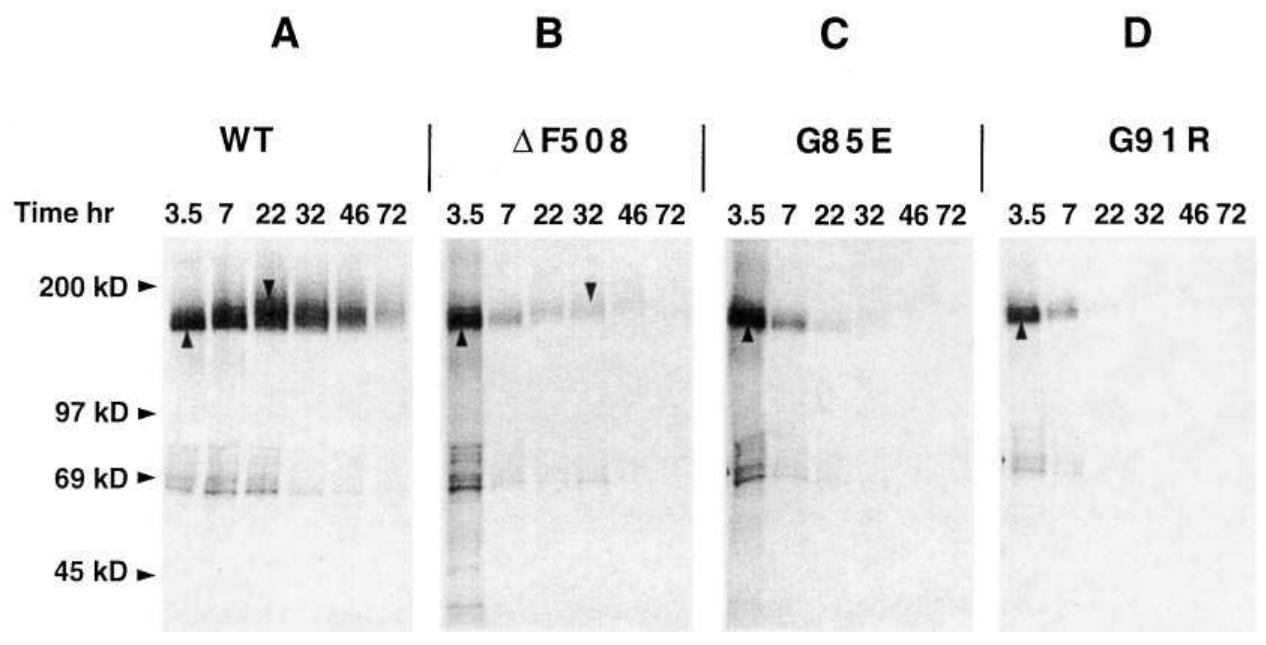

Figure 3. CFTR processing in $\mathrm{Xe}$ nopus oocytes. Microinjected Xenopus oocytes expressing WT, G85E, G91R, or $\triangle$ F508 CFTR were incubated in MBS for $3.5 \mathrm{~h}$, and were then placed in MBS containing 1 $\mathrm{mM}$ methionine. At indicated times groups of five oocytes were collected for immunoprecipitation and SDS-PAGE analysis. Full-length CFTR protein was identified by immunoprecipitation with $\mathrm{A}_{2}$ antibody as described. CFTR was initially recovered as a $150-\mathrm{kD}$ protein (upward arrows) which was processed to a more slowly migrating form of $165 \mathrm{kD}$ at later time points (downward arrows). 
amenable to topologic analysis. In addition, this system has been successfully used to define $\mathrm{NH}_{2}$ terminus topology of other polytopic proteins including the related ABC transporter MDR1 $(32,40)$.

Plasmids encoding WT, G85E, and G91R CFTR were truncated at codon $\mathrm{N} 186,48$ residues $\mathrm{COOH}$-terminal to TM2. Truncated plasmids were expressed in RRL in the presence of microsomal membranes, and newly synthesized chains were released from ribosomes with puromycin before digestion with $\mathrm{PK}$ in the presence and absence of nondenaturing detergent as described (32). Plasmids encoding WT protein generated polypeptides of the expected size $(22 \mathrm{kD}$; Fig. $4 A)$ which, after PK digestion, yielded a $17-\mathrm{kD}$ protease protected fragment (Fig. $4 A$, lane 2, upward arrow). This fragment was not observed in the presence of nondenaturing detergent (lane 3) nor in the absence of ER-derived microsomal membranes (data not shown). Thus, protease protection required an intact ER membrane, demonstrating that the chains contained both cytosolically accessible and lumenally protected polypeptide regions. The transmembrane topology of these truncated proteins was determined by immunoprecipitating translation products with antisera raised against synthetic peptides corresponding to residues no. 102-116, between TM1 and TM2 ( $\alpha-102$ antisera), or $\mathrm{NH}_{2}$-terminal residues no. 2-14 ( $\left.\alpha-5242\right)$. Both antisera were reactive with the $22-\mathrm{kD}$ fragments, as expected. The 17-kD protease-protected fragment, however, was recognized only with $\alpha-102$ antisera (Fig. 4 A, lanes 4-9). Residues $\mathrm{NH}_{2}$-terminal to TM1 were therefore accessible to PK, and were oriented towards the cytosol, while residues $\mathrm{COOH}$ terminal to TM1 resided in the ER lumen. These results confirmed that TM1 spanned the ER membrane in a C-trans (type II) topology, in agreement with current topologic models of CFTR $(3,41)$. To determine whether TM2 spanned the membrane in these truncated chains, we also analyzed an additional construct in which a passive $\mathrm{COOH}$ terminus translocation re- porter, P (derived from bovine prolactin [30]), was ligated COOH-terminal to TM2 at residue N186. In these chains, the $P$ reporter was quantitatively digested by exogenously added PK (Fig. 4 B). TM2 therefore spanned the membrane in an orientation opposite to that of TM1, with its $\mathrm{NH}_{2}$ terminus flanking sequences in the ER lumen, and $\mathrm{COOH}$ terminus flanking sequences in the cytosol.

The effect of G85E and G91R mutations on CFTR topology was similarly determined using plasmids TM1-2 (G85E) and TM1-2 (G91R). After expression in RRL and PK digestion, chains generated from these plasmids yielded protected fragments indistinguishable from WT CFTR (Fig., $5 A$ and $B$, lanes 1-3). Immunoprecipitation of proteolysis products with $\alpha-102$ and $\alpha-5242$ antisera confirmed that the $\mathrm{NH}_{2}$ and $\mathrm{COOH}$ termini of TM1 in these mutant chains were oriented in the same manner (cytosol and ER lumen, respectively) as WT chains. Because no other hydrophobic segments in this region of CFTR are of sufficient length to span the membrane (in helical conformation), these results demonstrate that mutant TM1 spanned the membrane, and suggest that charged residues $85 \mathrm{E}$ and $91 \mathrm{R}$ were located within the plane of the lipid bilayer. Because this technique does not precisely identify TM1 boundaries, however, we cannot formally discount the possibility that residues $85 \mathrm{E}$ or $91 \mathrm{R}$ were adjacent to the bilayer, particularly if TM1 spanned the membrane as a beta strand, as has been recently described for several COOH terminus TM segments of the GLAST-1 glutamate transporter (42). Despite these limitations, these studies demonstrate that G85E and G91R mutations do not disrupt CFTR topology in the ER membrane, and provide a reasonable two dimensional localization of the mutant residues relative to the lipid bilayer.

Disruption of CFTR trafficking is due to charge insertion into TM1. Results shown in Figs. 4 and 5 suggest that defective trafficking of G85E and G91R CFTR resulted from introduction of a charged residue within the lipid bilayer, but they
A

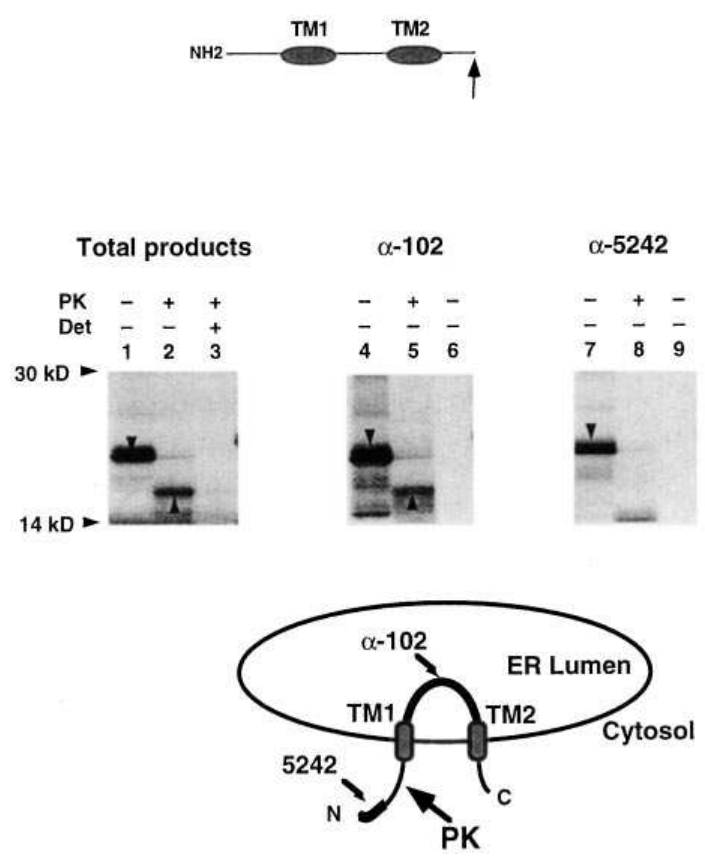

B
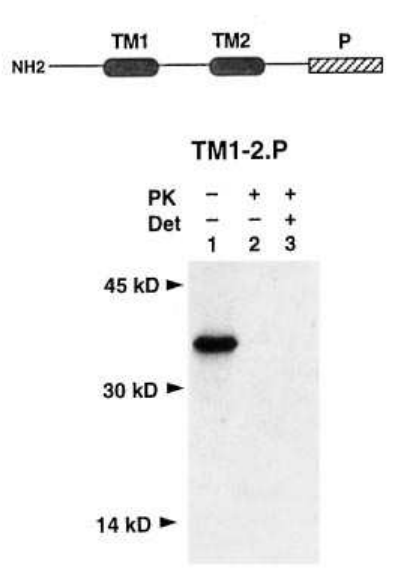

Figure 4. Transmembrane topology of WT TM1. $(A)$ WT CFTR cDNA was truncated at BP 565 in the coding sequence and translated in rabbit reticulocyte lysate in the presence of microsomal membranes. Translation products were digested with PK in the presence or absence of detergent (det) as indicated, and were analyzed directly by SDSPAGE (lanes 1-3). Translation products were immunoprecipitated with $\alpha-102$ antisera (lanes 4-6) or $\alpha-5242$, antisera (lane 7-9) before SDS-PAGE. Proteolysis of fulllength chains (downward arrows) generated a protected fragment (upward arrows) reactive only to $\alpha-102$ antisera. (B) Immunoprecipitation of proteolysis products from plasmid TM1-2.P using antiprolactin antisera. Topology of chains is schematically diagrammed; shaded ovals represent putative TM segments. 
B

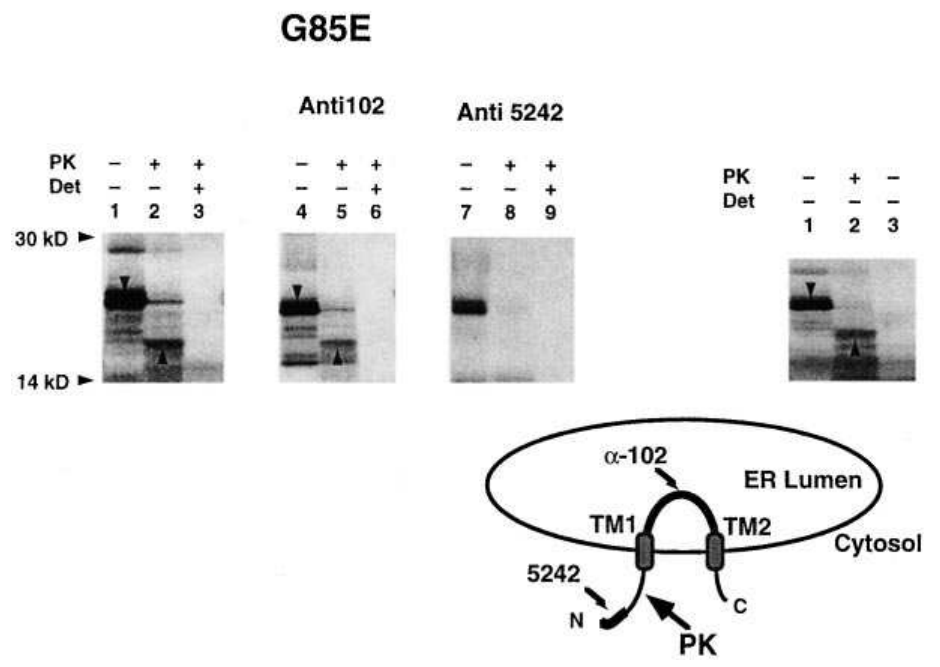

\section{G91R}

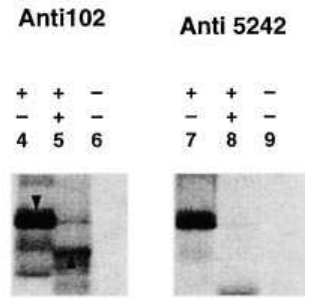

Figure 5. Transmembrane topology of mutant TM1. CFTR cDNAs encoding G85E and G91R mutations were truncated at BP 565, translated in RRL, and analyzed as described in Fig. 4. do not rule out the possibility that loss of a glycine residue within TM1 might instead be responsible. We therefore examined the effects of two additional (non CF-related) TM1 mutations, G91A and G91E. In Xenopus oocytes, full-length CFTR containing the conserved G91A mutation was stable and efficiently processed after microinjection of cRNA (Fig. $6 A$ ). In contrast, $\sim 70 \%$ of chains containing G91E were degraded within $30 \mathrm{~h}$ of injection, similar to results obtained using the G85E and G91R constructs. In addition, neither the alanine nor glutamic acid substitutions at position 91 significantly affected $\mathrm{NH}_{2}$ terminus topology (Fig. $6 \mathrm{~B}$ ). Although it is possible that the G91E and G91R mutations might have different effects on CFTR structure, the similar effects of acidic as well as basic residues on CFTR trafficking, and the lack of effect of the conservative alanine substitution, all support the conclusion that CFTR is highly sensitive to insertion of an additional charged residue within the hydrophobic segment, TM1.

Structural requirements for CFTR stability. Our observations indicate that a mutant-charged residue within TM1 in some manner provides structural cues for ER degradation machinery, but they do not distinguish whether such residues are recognized directly or whether they exert indirect effects by alter- ing other aspects of CFTR structure. We reasoned that if charged residues $85 \mathrm{E}$ or $91 \mathrm{R}$ were recognized directly within the bilayer, then ER quality control machinery might preferentially degrade mutant chains at a relatively early stage of synthesis, for example after synthesis of only one or two TM segments. If, however, these mutations disrupted more global aspects of CFTR tertiary structure, then discrimination of mutant and WT chains might require synthesis of a large portion of CFTR. To sort out these possibilities, we therefore attempted to identify when during CFTR synthesis cellular quality control machinery was first able to distinguish mutant from WT chains.

Termination codons were engineered at residues 188, 393, 589, and 836 (COOH terminus to TM2, TM6, NBD1, and the $\mathrm{R}$ domain, respectively) in WT, G85E, and G91R CFTR. When plasmids terminated after TM2 or TM6 were expressed in pulse-chase labeled oocytes, we found both WT and mutant chains were degraded within $24 \mathrm{~h}$ (Fig. 7). Thus, the first two TM segments as well as the entire $\mathrm{NH}_{2}$ terminus hydrophobic domain (TMD1) of CFTR were insufficient to generate a stable protein. A lag in degradation of 393X chains at $6 \mathrm{~h}$ (Fig. 7 $B$ ) was likely due to labeling conditions in this experiment, as this was not observed for all batches of oocytes studied. G85E
A

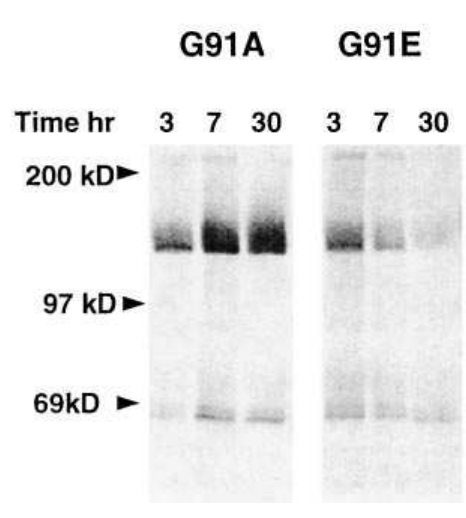

B

G91A

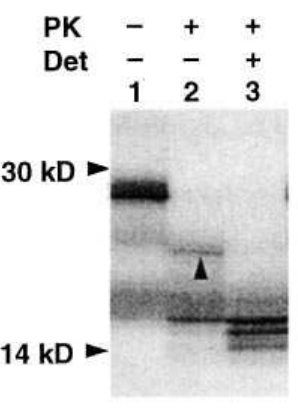

Figure 6. Trafficking and topology of G91A and G91E CFTR. $(A)$ Full-length CFTR encoding mutations G91A or G91E was transcribed and injected into oocytes as described in Fig. 2. Oocytes were moved to media containing $1 \mathrm{mM}$ methionine $3 \mathrm{~h}$ after injection, and were collected for immunoprecipitation at the times indicated. $(B)$ G91A and G91E CFTR cDNAs were truncated at BP 565, transcribed, and expressed in RRL as described in Fig. 3. Shown are total translation products before and after PK digestion as indicated. $U p$ ward arrow indicates protease-protected fragment. 
A

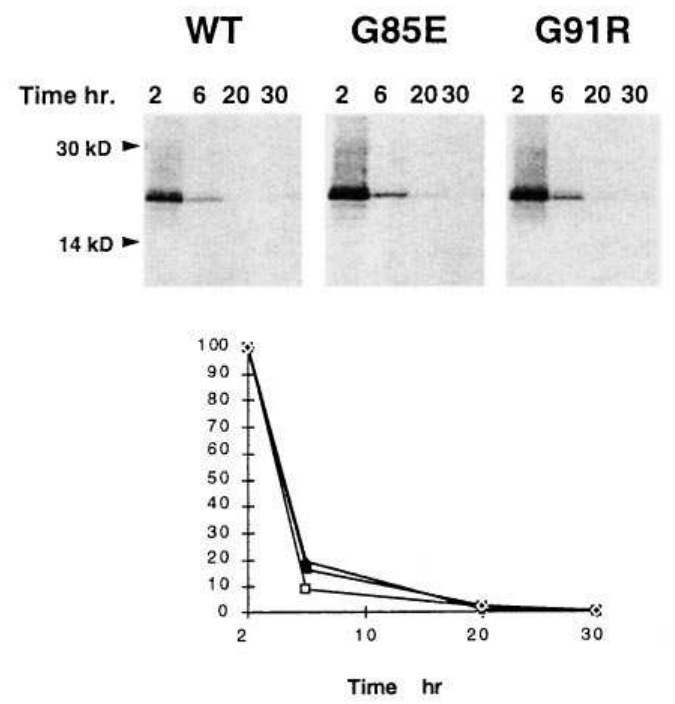

C

WT

G85E

$\begin{array}{llll}3 & 7 & 24 & 32 \\ 48\end{array}$

$\begin{array}{lllll}3 & 7 & 24 & 32 & 48\end{array}$
G91R

$\begin{array}{llll}3 & 7 & 24 & 32 \\ 48\end{array}$

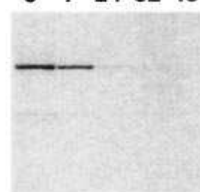

B

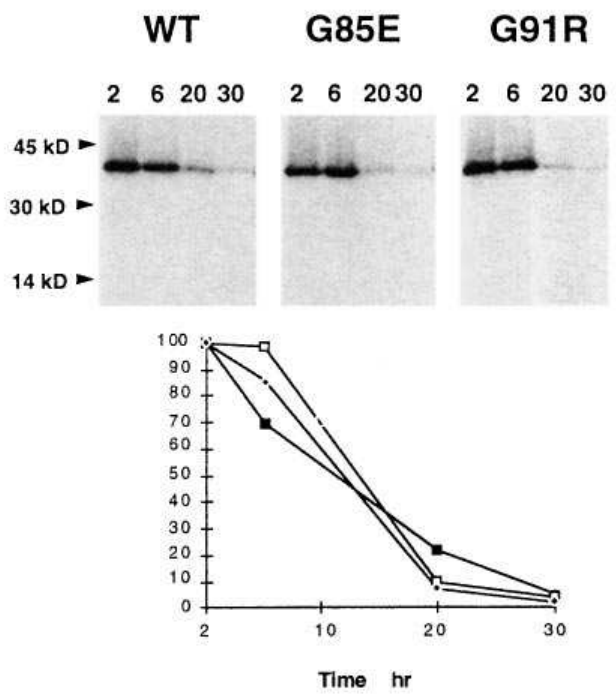

D
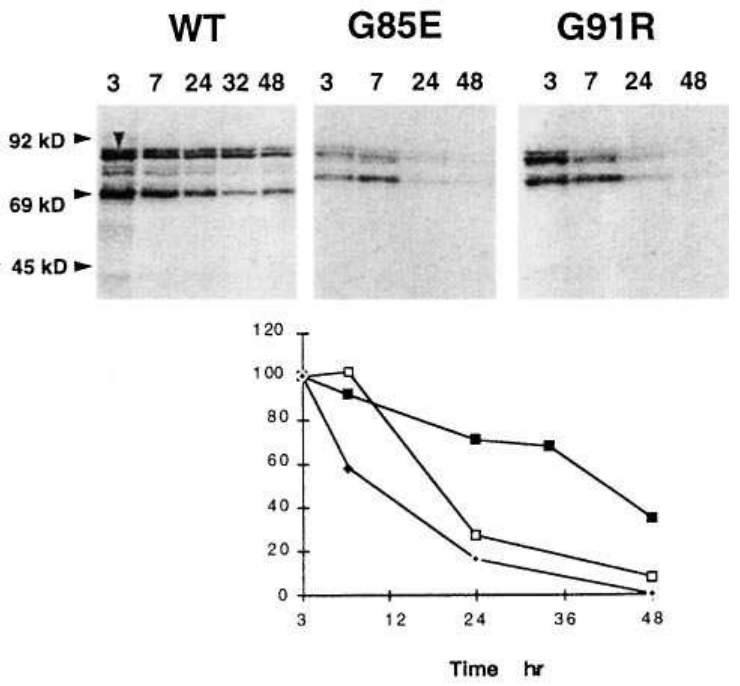

Figure 7. Degradation of truncated WT and mutant CFTR in oocytes. CFTR chains were expressed in microinjected pulse chase-labeled oocytes as described in Fig. 2. Chains were truncated by engineering termination codons at residues $188(A), 393(B), 589(C)$ and $836(D)$. In $D$, the upper band (downward arrow) migrates as a doublet because of phosphorylation (data not shown), and is the expected size for these truncated chains. The identity of the lower bands is unknown, but may represent translation initiation at an internal ATG site, or possibly degradation products. Quantitation of autoradiograms is shown. Filled squares, WT; open squares, G85E; closed diamonds, G91R.

and G91R mutant proteins truncated after NBD1 or the R domain were also rapidly degraded (80\% of chains) within $24 \mathrm{~h}$. $40 \%$ of WT 589 X and nearly $80 \%$ of WT 836 X CFTR, however, achieved a stable conformation that was present in cells 24-32 $\mathrm{h}$ after injection.

\section{Discussion}

It is now generally appreciated that most, if not all eukaryotic cells, exhibit a quality control system in the ER whose function is to degrade proteins that fail to fold or assemble properly (43,
44). In this study, we show that Xenopus oocytes also exhibit a quality control system that is capable of distinguishing mutant from WT CFTR proteins. Although the cellular machinery involved in this process is currently unknown, oocytes selectively targeted $\Delta \mathrm{F} 508, \mathrm{G} 85 \mathrm{E}$, and G91R CFTR chains into a preGolgi, rapid degradation pathway, while WT CFTR efficiently exited the ER and was processed in the Golgi complex. Stability and intracellular processing of full-length WT and mutant CFTR proteins correlated well with the degree of reduction in functional plasma membrane chloride channel activity, suggesting that as in mammalian cells, protein folding and ER 
quality control in oocytes play a key role in the disruption of CFTR by these mutations. Our studies therefore demonstrate that Xenopus oocytes, which have been widely used for examining aspects of CFTR ion channel function, also provide a versatile system for studying cellular processes that govern intracellular trafficking and degradation of mutant CFTR proteins.

While CFTR processing in Xenopus oocytes exhibited many key features previously reported in mammalian cells, several differences were noted. Synthesis of full-length CFTR required $90 \mathrm{~min}$, and newly synthesized chains resided in the ER for $24 \mathrm{~h}$ or more before trafficking through the Golgi complex. In contrast, synthesis of CFTR in mammalian cells required less than $15 \mathrm{~min}$, and exit of CFTR from the ER was essentially complete within 3-4 h (15). The slower kinetics observed in oocytes appeared to be largely due to the reduced incubation temperature $\left(18^{\circ} \mathrm{C}\right)$, because when microinjected oocytes were incubated at $30-37^{\circ} \mathrm{C}$, rates of CFTR synthesis and processing approached that of mammalian cells (A. Bragin and W. Skach, unpublished observation). At these high temperatures, however, oocyte viability was markedly compromised.

Several studies have reported that $80 \%$ of heterologously expressed WT CFTR is degraded in the ER of mammalian cells $(13,15,17)$. In oocytes, however, up to $100 \%$ of WT CFTR was stable and processed by Golgi enzymes. In addition, although $\Delta \mathrm{F} 508, \mathrm{G} 85 \mathrm{E}$, and G91R mutants exhibited a dramatic defect in ER trafficking, a small but significant fraction of these chains appeared to reach the oocyte plasma membrane, most likely accounting for the low levels of chloride efflux observed by us and others (37). An interesting question regarding these results is whether the increased efficiency of CFTR processing in oocytes reflects $(a)$ a lower threshold of oocyte quality control machinery in discriminating mature from misfolded CFTR; $(b)$ qualitative differences in oocyte folding machinery that promote more efficient CFTR maturation; or $(c)$ whether CFTR proteins simply mature more efficiently at reduced temperatures. Studies examining degradation mechanisms of CFTR synthesized in different expression systems may shed insight into these possibilities.

In the current study we observed that the trafficking efficiency of G85E and G91R CFTR out of the ER in oocytes was similar to or slightly less than that of $\Delta$ F508 CFTR. Unlike $\Delta \mathrm{F} 508$, however, G85E and G91R mutations have been associated with a mild disease phenotype in humans $(33,34,45)$. While the reason for these different disease phenotypes is unknown, it is possible that subtle differences in CFTR folding and/or ER quality control may exist in mammalian cells, which accounts for the differences in disease severity. Alternatively, it is possible that the different clinical phenotypes result from specific aspects of CFTR function that are disrupted by mutations in different regions of the protein.

Based on our previous studies of human MDR1 biogenesis $(32,40,46)$, we predicted that the introduction of a charged residue into TM1 of CFTR would interfere with ability of TM1 to function as an internal signal sequence, and direct $\mathrm{NH}_{2}$ terminus assembly into the ER membrane. Surprisingly, while G85E and G91R mutations each disrupted TM1 signal sequence activity (data not shown), neither mutation affected TM1 transmembrane topology. Instead, peptide translocation of G85E and G91R CFTR was initiated by a downstream signal sequence, TM2, which functioned to establish $\mathrm{NH}_{2}$ termi- nus topology through a novel, posttranslational mechanism (W. Skach, unpublished observations). Like MDR1, CFTR therefore appears to exhibit two redundant mechanisms for $\mathrm{NH}_{2}$ terminus transmembrane assembly that use signal sequence activities encoded in both TM1 and TM2. In the case of CFTR, however, these redundant mechanisms are required for transmembrane assembly because two charged residues within TM1, E92, and K95, impair intrinsic TM1 signal sequence activity. Furthermore, it is this redundant signal sequence activity of TM2 that is responsible for directing proper CFTR $\mathrm{NH}_{2}$ terminus topology in the G85E and G91R mutants (W. Skach, unpublished observations). These results are consistent with findings of Carroll et al., where it was shown that $\mathrm{NH}_{2}$ terminus deletions did not abolish CFTR chloride channel activity (47), presumably because the deletion of TM1 would likely have little effect on the independent topogenic activity (or topology) of remaining TM segments. In this case, assembly of CFTR chains lacking TM1 appears to be sufficient for generating detectable chloride channel activity, while mutations within TM1 may disrupt CFTR function through other aspects of protein folding and/or processing.

Charged residues within TM segments have been implicated in influencing intracellular trafficking, degradation, and oligomerization of single-spanning or bitopic proteins (48-51). Remarkably however, CFTR already contains charged residues in predicted TM segments no. 1, 2, 6, 7, 8, 9, 10, and 11 that likely facilitate interactions between TM helices and/or contribute to the structure of the chloride channel pore $(6,52)$. In this regard, cysteine-scanning mutagenesis has indicated that residues G85 and G91 are both accessible to aqueous sulfhydryl-reactive reagents, and thus are proposed to lie in an aqueous environment, rather than in direct physical contact with lipid (52). While mutant residues $85 \mathrm{E}$ or $91 \mathrm{R}$ appear to be localized within the plane of the bilayer, it is likely that they may also reside in contact with solvent rather than in direct contact with membrane lipids per se.

One way to view CFTR maturation is to consider that all CFTR molecules initially start out in an unfolded, transportincompetent state, and that proper maturation involves acquisition of specific structure that is no longer recognized by quality control machinery. Our data support this view, and demonstrate that truncated fragments of CFTR were rapidly degraded in cells until synthesis of at least NBD1 had been completed. Consistent with this, cells were also unable to distinguish structural perturbations induced by G85E or G91R mutations until synthesis of NBD1. Because mutant residues were localized within or adjacent to the bilayer, the simplest interpretation is that residues $85 \mathrm{E}$ or $91 \mathrm{R}$ might cause a subtle alteration in helical packing and/or conformation of cytosolic/ extracytosolic connecting peptide loops. Although these techniques do not allow us to define the precise nature of this structural alteration, we predict that this alteration results in a change in CFTR tertiary structure either within TMD1 or between TMD1 and other CFTR domains, preventing proper maturation and resulting in recognition of chains by ER quality control machinery.

Finally, our results also indicate that only after synthesis of the R domain do truncated CFTR chains exhibit intracellular stability resembling that of full-length protein. These results agree with prior observations that the D836X truncation generated functional cAMP-mediated chloride channels in mammalian cells (53). The chloride conductance of oocytes ex- 
pressing the D836X mutant, however, was significantly less than oocytes expressing full-length CFTR (K. Foskett and W. Skach, unpublished observations). Thus, while failure of CFTR to mature in the ER as determined by degradation of $\Delta F 508$, CFTR does not predict whether or not CFTR is able to acquire chloride channel function (14), maturation in the ER as determined by stability of D836X chains also does not appear to correlate with different structural requirements for later trafficking events and/or ion channel function. An important interpretation of these observations is that structural features of polytopic proteins required for specific functions may be distinct from features required for normal intracellular trafficking and visa versa. Understanding how polytopic proteins accomplish these two different aspects of folding will provide important insight into our view of diseases such as $\mathrm{CF}$, where these two processes are uncoupled.

\section{Acknowledgments}

The authors thank Mr. Kenneth Moss and Mr. Andrew Helm for technical assistance, and Dr. Kevin Foskett for helpful comments in reviewing the manuscript. The authors also thank Drs. Monique Mansoura and David Dawson for providing plasmids pBQ4.7 G91A and pBQ4.7 G91E.

This work was supported by grants from the Cystic Fibrosis Foundation, and the National Institutes of Health (CA01614 and DK51818)

\section{References}

1. Riordan, J.R., J.M. Rommens, B.-S. Kerem, N. Alon, R. Rozmahel, Z. Grzelczak, J. Zielenski, S. Lok, F.S. Collins, and L.-C. Tsui. 1989. Identification of the cystic fibrosis gene: cloning and characterization of complementary DNA. Science (Wash. DC). 245:1066-1072.

2. Marino, C., L. Matovich, F. Goerlick, and J. Cohn. 1991. Localization of the cystic fibrosis transmembrane conuctance regulator in the pancreas. J. Clin. Invest. 88:712-716.

3. Denning, G., L. Ostedgaard, S. Cheng, A. Smith, and M. Welsh. 1992. Localization of cystic fibrosis transmembrane conductance regulator in chloride secretory epithelia. J. Clin. Invest. 89:339-349.

4. Higgins, C. 1992. ABC transporters: from microorganisms to man. Ann. Rev. Cell Biol. 8:67-113.

5. Hyde, S.C., P. Emsley, M.J. Hartshorn, M.M. Mimmack, U. Gileadi, S.R. Pearce, M.P. Gallagher, D.R. Gill, R.E. Hubbard, and C.F. Higgins. 1990. Structural model of ATP-binding proteins associated with cystic fibrosis, multidrug resistance and bacterial transport. Nature (Lond.). 346:362-365.

6. Anderson, M.P., R.J. Gregory, S. Thompson, D.W. Souza, S. Paul, R.C. Mulligan, A.E. Smith, and M.J. Welsh. 1991. Demonstration that CFTR is a chloride channel by alteration of its anion selectivity. Science (Wash. DC). 253: 202-204.

7. Hasegawa, H., W. Skach, O. Baker, M.C. Cayalag, V. Lingappa, and A. Verkman. 1992. A multifunctional aqueous channel formed by CFTR. Science (Wash. DC). 258:1477-1479.

8. Reisin, I., A. Prat, E. Abraham, J. Amara, R. Gregory, D. Ausiello, and H. Cantiello. 1994. The cystic fibrosis transmembrane conductance regulator is a dual ATP and chloride channel. J. Biol. Chem. 269:20584-20691.

9. Ohrui, T., W. Skach, M. Thompson, J. Matsumoto-Pon, M.C. Calayag, and J. Willicombe. 1994. Radiotracer studies of CFTR expressed in Xenopus oocytes. Am. J. Physiol. (Cell. Physiol.) C1586-C1593.

10. Cystic Fibrosis Genetic Analysis Consortium. 1997. Cystic fibrosis mutation data base. www.genet.sickkids.on.ca/cftr

11. Welsh, M., and A. Smith. 1993. Molecular mechanisn of CFTR chloride channel dysfunction in cystic fibrosis. Cell. 73:1251-1254.

12. Denning, G., M. Anderson, J. Amara, J. Marshall, A. Smith, and M. Welsh. 1992. Processing of mutant cytsic fibrosis transmembrane conductance regulator is temperature-sensitive. Nature (Lond.). 358:761-763.

13. Cheng, S.H., R.J. Gregory, J. Marshall, S. Paul, D.W. Souza, G.A. White, C.R. O'Riordan, and A.E. Smith. 1990. Defective intracellular transport and processing on CFTR is the molecular basis of most cystic fibrosis. Cell. 63 : 827-834

14. Pasyk, E., and K. Foskett. 1995. Mutant ( $\Delta \mathrm{F} 508)$ cystic fibrosis transmembrane conductance regulator $\mathrm{Cl}^{-}$channel is functional when retained in the endoplasmic reticulum of mammalian cells. J. Biol. Chem. 270:12347-12350.
15. Ward, C., and R. Kopito. 1994. Intracellular turnover of cystic fibrosis transmembrane conductance regulator. J. Biol. Chem. 269:25710-25718.

16. Ward, C., C. Omura, and R. Kopito. 1995. Degradation of CFTR by the ubiquitin-proteosome pathway. Cell. 83:121-128.

17. Jensen, T., M. Loo, S. Pind, D. Williams, A. Goldberg, and J. Riordan. 1995. Multiple proteolytic systems, including the proteosome, contribute to CFTR processing. Cell. 83:129-136.

18. Lukacs, G., A. Mohamed, N. Kartner, X. Chang, J. Riordan, and S Grinstein. 1994. Conformational maturation of CFTR but not its mutant counterpart (delta F508) occurs in the endoplasmic reticulum and requires ATP. EMBO J. 13:6076-6086.

19. Yang, Y., S. Janach, J. Cohn, and J. Wilson. 1993. The common variant of cystic fibrosis transmembrane conductance regulator is recognized by hsp70 and degraded in a pre-Golgi nonlysosomal compartment. Proc. Nat. Acad. Sci. USA. 90:9480-9484.

20. Pind, S., J. Riordan, and D. Williams. 1994. Participation of the endoplasmic reticulum chaperone calnexin (p88, IP90) in the biogenesis of the cystic fibrosis transmembrane conductance regulator. J. Biol. Chem. 269:1278412788.

21. Brown, C.R., L. Hong-Brown, J. Biwersi, A. Verkman, and W. Welch. 1996. Chemical chaperones correct the mutant phenotype of the $\Delta$ F508 cystic fibrosis transmembrane conductance regulator protein. Cell Stress and Chaperones. 1:117-125

22. Sato, S., C. Ward, M. Krouse, J. Wine, and R. Kopito. 1996. Glycerol reverses the misfolding phenotype of the most common cystic fibrosis mutation. $J$. Biol. Chem. 271:635-638.

23. Thomas, P., P. Shenbagamurthi, J. Sondek, J. Hullihen, and P. Pederson. 1992. The cystic fibrosis transmembrane conductance regulator. Effects of the most common cystic fibrosis-causing mutation on the secondary structure and stability of a synthetic peptide. J. Biol. Chem. 267:5727-5730.

24. Hartman, J., Z. Huang, T. Rado, S. Peng, T. Jiling, D. Muccio, and E. Sorscher. 1992. Recombinant synthesis, purification, and nucleotide binding characteristics of the first nucleotide binding domain of the cystic fibrosis gene product. J. Biol. Chem. 267:6455-6458.

25. Blobel, G. 1980. Intracellular protein topogenesis. Proc. Natl. Acad. Sci. USA. 77:1496-1500.

26. Bibi, E., G. Verner, C.-Y. Chang, and H. Kaback. 1991. Organization and stability of a polytopic membrane protein: deletion and analysis of the lactose permease of Escherichia coli. Proc. Natl. Acad. Sci. USA. 88:7271-7275.

27. Bibi, E., S. Stearns, and H. Kaback. 1992. The N-terminal 22 amino acid residues in the lactose permease of Escherichia coli are not obligatory for membrane insertion or transport activity. Proc. Natl. Acad. Sci. USA. 89:3180-3184.

28. Calamia, J., and C. Manoil. 1992. Membrane protein spanning segments as export signals. J. Mol. Biol. 224:539-543.

29. Skach, W., and V. Lingappa, 1993. Intracellular trafficking of pre(pro-) proteins across RER membranes. In Mechanisms of Intracellular Trafficking and Processing of Pro-proteins. Y.P. Loh, editor. CRC Press, Inc., Boca Raton, FL. 19-77.

30. Rothman, R.E., D.W. Andrews, M.C. Calayag, and V.R. Lingappa. 1988. Construction of defined polytopic integral transmembrane proteins. $J$. Biol. Chem. 263:10470-10480.

31. Hartmann, E., T.A. Rapoport, and H.F. Lodish. 1989. Predicting the orientation of eukaryotic membrane-spanning proteins. Proc. Natl. Acad. Sci. USA. 86:5786-5790.

32. Skach, W., and V. Lingappa. 1993. Amino terminus assembly of human P-glycoprotein at the endoplasmic reticulum is directed by cooperative actions of two internal sequences. J. Biol. Chem. 268:23552-23561.

33. Guillermit, H., M. Jehanne, I. Quere, M. Audrezet, B. Mercier, and C. Ferec. 1993. A novel mutation in exon 3 of the CFTR gene. Hum. Genet. 91: 233-235.

34. Zielenski, J., D. Bozon, B. Kerem, D. Markiewicz, P. Durie, J.M. Rommens, and L.-C. Tsui. 1991. Identification of Mutations in exons 1 through 8 of the cystic fibrosis transmembrane conductance regulator (CFTR) gene. $\mathrm{Ge}$ nomics. 10:229-235.

35. Barth, L.G., and L.J. Barth. 1959. Differentiation of cells of the Rana pipiens gastrula in unconditioned medium. J. Embryol. Exp. Morphol. 7:210 222 .

36. Cohn, J., A. Narin, C. Marino, O. Melhus, and J. Kole. 1992. Characterization of the cystic fibrosis transmembrane conductance regulator in a colonocyte cell line. Proc. Natl. Acad. Sci. USA. 89:2340-2344.

37. Drumm, M., D. Wilkenson, L. Smit, R. Woeerll, T. Strong, R. Frizzell, D. Dawson, and F. Collins. 1991. Chloride conductance expressed by $\Delta$ F508 and other mutant CFTRs in Xenopus oocytes. Science (Wash. DC). 254:17971799.

38. Bear, C., F. Duguay, A. Naismith, N. Kartner, J. Hanrahan, and J. Riordan. 1991. CL- channel activity in Xenopus oocytes expressing the cystic fibrosis gene. J. Biol. Chem. 266:19142-19145.

39. Walter, P., and G. Blobel. 1983. Preparation of microsomal membranes for cotranslational protein purification. In Methods in Enzymology. Vol. 96. S. Fleischer and B. Fleischer, editors. Academic Press, Inc., New York. 84-93.

40. Skach, W., and V. Lingappa. 1994. Transmembrane orientation and topogenesis of the 3rd and 4th membrane-spanning regions of human P-glycopro- 
tein (MDR1). Cancer Res. 54:3202-3209.

41. Chang, X.-B., Y.-X. Hou, T. Jensen, and J. Riordan. 1994. Mapping of cystic fibrosis transmembrane conductance regulator membrane topology by glycosylation site insertion. J. Biol. Chem. 269:18572-18575.

42. Wahle, S., and W. Stoffel. 1996. Membrane topology of the high-affinity L-glutamate transporter (GLAST-1) of the central nervous system. J. Cell Biol. 135:1867-1877.

43. Klausner, R., and R. Sita. 1990. Protein degredation in the endoplasmic reticulum. Cell. 62:611-614.

44. Lippincott-Schwartz, J., J. Bonifacino, L. Yuan, and R. Klausner. 1988. Degredation from the endoplasmic reticulum: disposing of newly synthesized proteins. Cell. 54:209-220.

45. Chalkley, G., and A. Harris. 1991. A cystic fibrosis patient who is homozygous for the G85E mutation has very mild disease. J. Med. Genet. 28:875877.

46. Skach, W., M.C. Calayag, and V. Lingappa. 1993. Evidence for an alternate model of human P-glycoprotein structure and biogenesis. J. Biol. Chem. 268:6903-6908.

47. Carroll, T., M. Morales, S. Fulmer, S. Allen, T. Flotte, G. Cutting, and W. Guggino. 1995. Alternate translation initiation codons can create functional forms of cystic fibrosis transmembrane conductance regulator. J. Biol. Chem. 270:11941-11946.

48. Adams, G.A., and J.K. Rose. 1985. Incorporation of a charged amino acid into the membrane-spanning domain blocks cell surface transport but not membrane anchoring of a viral glycoprotein. Mol. Cell. Biol. 5:1442-1448.

49. Davis, G.L., and E. Hunter. 1987. A charged amino acid substitution within the transmembrane anchor of the rous sarcoma virus envelope glycoprotein affects surface expression but not intracellular transport. J. Cell Biol. 105: 1191-1203.

50. Bonifacino, J., C. Suzuki, and R. Klausner. 1990. A peptide sequence confers retention and rapid degradation in the endoplasmic reticulum. Science (Wash. DC). 247:79-82.

51. Bonifacino, J., P. Cosson, and R. Klausner. 1990. Colocalized transmembrane determinants for ER degradation and subunit assembly explain the intracellular fate of TCR chains. Cell. 63:503-513.

52. Akabas, M., C. Kaufmann, A. Cook, and P. Archdeacon. 1994. Amino acid residues lining the chloride channel of the cystic fibrosis transmembrane conductance regulator. J. Biol. Chem. 269:14865-14868.

53. Sheppard, D., L. Ostedgaard, P. Rich, and M. Welsh. 1994. The aminoterminal portion of CFTR forms a regulated $\mathrm{Cl}^{-}$channel. Cell. 76:1091-1098. 